\title{
Antimicrobial susceptibility pattern and serotyping of Streptococcus pneumoniae isolated from Kanti Children Hospital in Nepal
}

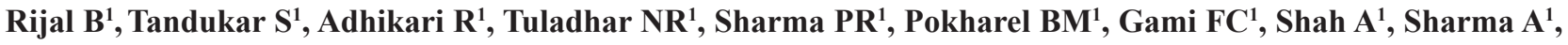

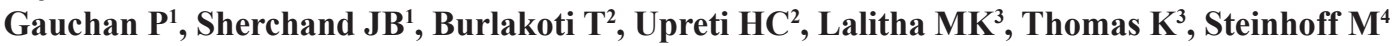 \\ ${ }^{1}$ Institute of Medicine, Maharajganj, Kathmandu, Nepal, ${ }^{2}$ Kanti Children Hospital, Kathmandu, Nepal, ${ }^{3}$ Christian \\ Medical College, Vellore, India, ${ }^{4}$ Johns Hopkins University, School of Public Health, Baltimore, USA.
}

\begin{abstract}
Background: Invasive pneumococcal disease is a significant cause of morbidity and mortality worldwide and it is a major cause for childhood deaths in Nepal.

Objectives: The aim of this study was to establish the antimicrobial susceptibility pattern of Streptococcus pneumoniae and perform serotype responsible for pneumococcal disease in Nepal.

Materials and methods: All together 3774 children from 2 to 60 months who fulfilled the enrollment criteria for suspect of bacterial pneumonia, sepsis or meningitis were enrolled for etiologic studies of severe illness. During the study period 60 isolates of Streptococcus pneumoniae were isolated and the antimicrobial susceptibility testing and serotyping were performed.

Results: The study showed that $24(52.17 \%)$ isolates were resistant to Cotrimoxazole, $3(6.5 \%)$ isolates were intermediately resistant to Penicillin but no Penicillin resistant strains were isolated. The $1(2.17 \%)$ isolate was recorded as Erythromycin and Chloramphenicol resistant and only $1(2.17 \%)$ isolate was found intermediately resistant to Cefotaxime. Of the 60 isolates, serotyping result was available only for 46 isolates. The most common serotypes were serotype $1(27.65 \%)$ followed by serotype $5(19.14 \%)$ and serotype $4(8.5 \%)$ respectively followed by serotype $39,23 \mathrm{~F}$, 7F, 19B, 12A, 14, 18F, 6B, 32, 16, 19F and 25F.

Conclusions: Alarming level of Cotrimoxazole resistance demands revision of pneumonia treatment policy in Nepal and rising tendency of other drug resistance against Streptococcus pneumoniae showed use of these drugs for the treatment of meningitis, pneumonia and other serious infections needs extended research. The common serotype 1, 5 and 4 need to be incorporated in pneumococcal vaccine to immunise children in Nepal.
\end{abstract}

Key words: Antimicrobial susceptibility, Streptococcus pneumoniae, Serotyping, Nepal

$\mathrm{T}^{\mathrm{s}}$ he serious pneumococcal infections such as pneumonia, bacteraemia, meningitis, and otitis media remain major causes of morbidity and mortality in persons of all age's worldwide ${ }^{1,2,3}$. The ability of pneumococci to cause disease is directly related to the production of a capsule, a polysaccharide structure external to the cell wall that provides resistance to phagocytosis and promotes evasion of the host immune system by the bacteria ${ }^{4}$. While attack rates for pneumococcal disease are extremely high in developing countries, indigenous and disadvantaged minorities in developed countries are also at particular risk ${ }^{2}$. The emergence of drug-resistant Streptococcus pneumoniae will make these common infections more difficult to treat ${ }^{5}$, as isolates resistant to one or more first-line agents are common in many parts of the world ${ }^{6}$. Since the first report of decreased susceptibility to penicillin in 1967, resistance of Streptococcus pneumoniae to this drug, as well as other antibiotics, has been reported in many countries ${ }^{2}$. Resistant pneumococci may spread from country to country, and widened and intensified surveillance is needed in all areas, including those countries where resistance is relatively uncommon ${ }^{7}$.

Invasive pneumococcal disease is estimated to cause more than 50, 000 cases of bacteraemia and 3,000-5, 000 cases of meningitis annually. Death due to invasive disease is estimated to be 6,000 persons annually, with more than half of these in adults with specific risk factors (age, immunosuppression) for severe disease. Such risk factors are an indication for vaccination ${ }^{8}$. Apart from

\footnotetext{
Correspondence

Dr. Basista Rijal

Department of Microbiology

Medical Campus and Tribhuvan University Teaching Hospital

Maharajganj, Kathmandu, Nepal

E-mail: rijal_basista@hotmail.com
} 
epidemic, at least 1.2 million cases of meningitis are estimated to occur every year with 135, 000 deaths ${ }^{9}$.

The goal of this study was to show the antimicrobial resistance trends and to find out the distribution of serotypes in invasive isolates of Streptococcus pneumoniae in Nepal.

\section{Materials and methods}

Study period and study site: In Nepal, an enhanced invasive pneumococcal disease surveillance programme began in November 2004 and data has been included up to December 2008.

Bacterial isolates: Pneumococci from patients with invasive pneumococcal diseases were isolated and identified in Kanti children Hospital. Blood and CSF including other body fluid were drawn and cultured to isolate the etiological agent following WHO procedure manual. Pneumococci from 3774 suspected children of 2 months to 60 months were isolated from blood, cerebrospinal fluid and pleural fluid during the 4 years study period.

Identification, antimicrobial susceptibility test and serotyping: Pneumococci were identified by Gram stain, colonial morphology on blood agar, and optochin susceptibility and were typed by the quelling reaction with antisera raised at Statens Seruminstitut. The tube dilution method was used to determine the Minimum inhibitory concentrations (MICs) for isolates of penicillin, Cefotaxime, Chloramphenicol, Erythromycin, and Cotrimoxazole. Susceptibility testing was conducted by (MIC determination by broth microdilution according to National Committee for Clinical Laboratory Standards (NCCLS) recommended procedures ${ }^{10}$. All isolates were tested against Penicillin, Erythromycin, Cotrimoxazole, Chloramphenicol and Cefotaxime. Quality control organisms specified by NCCLS, including S. pneumoniae ATCC (American type culture collection) 49619 , were used ${ }^{11}$. The proportion of isolates that were susceptible, intermediate and resistant to each agent was determined for the agents tested based on NCCLS interpretive criteria ${ }^{12}$. Intermediate and resistant strains were grouped as non susceptible for some analyses. Multidrug resistant (MDR) strains were defined as being non susceptible to all three drug classes tested. Serotyping was performed by capsular swelling reaction using group- and type-specific antisera (CDC produced or obtained from Statenserum Institute, Copenhagen, Denmark).

\section{Results}

A total of 60 clinical isolates of Streptococcus pneumoniae was recovered, of which 37 isolates were from blood, 18 from CSF and 5 from other body fluid.

Of the 3774 pneumonia suspected patients only 37 $(0.98 \%)$ patients showed culture positive, similarly of the 408 suspects of meningitis and CSF was cultured, only $18(4.4 \%)$ were culture positive for Streptococcus pneumoniae and out of 15 pleural fluid from the patients suspected of pneumonia only $5(33 \%)$ were culture positive as shown in fig 1 .

Overall 15 most common serotypes was detected in order of prevalence, being serotype 1 (27.65\%), followed by serotype 5 (19.14\%), 4 (8.5\%), 39 (4.25\%), 23F (4.25\%), 7F (4.25\%), 19B (4.25\%), 12A (4.25\%), $14(4.25 \%), 18 \mathrm{~F}(2.12 \%), 6 \mathrm{~B}(2.12 \%), 32(2.12 \%), 16$ (2.12\%), 19F (2.12\%) and $25 \mathrm{~F}$ and (2.12\%) (fig. 2).

Figure 3 shows the sensitivity of the pneumococcus isolates from the specimen. Serotype 1 was by far most common serotypes in each. MICs against penicillin, Cotrimoxazole, Chloramphenicol, Erythromycin and Cefotaxime were determined (Fig 3). Overall, there were $24(52.17 \%)$ isolates were resistance towards Cotrimoxazole and $9(19.56 \%)$ were considered intermediately resistant. Whereas penicillin MICs were mostly around $0.016 \mathrm{mgl}^{-1}$ and showed $3(6.5 \%)$ were intermediately resistant, no penicillin resistant strains were isolated. Over the 4 years, erythromycin MICs ranged from 0.062 to $8 \mathrm{mgl}^{-1}$ resistance was recorded in $1(2.17 \%)$. Chloramphenicol MICs ranged from 1 to $4 \mathrm{mgl}^{-1}$ over all $1(2.17 \%)$ was considered resistant toward Chloramphenicol where as the MICs of Cefotaxime ranges from $0.008 \mathrm{mgl}^{-1}$ to $0.25 \mathrm{mgl}^{-1}$. Overall only $1(2.17 \%)$ was found intermediately resistant to Cefotaxime in the study period. 


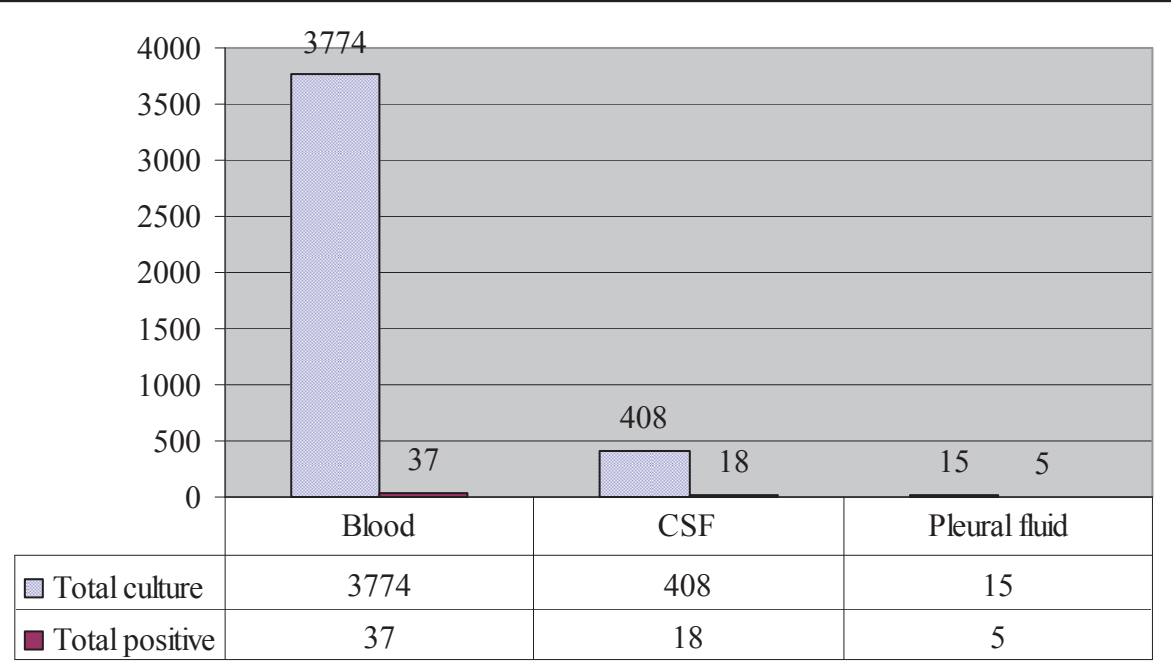

Fig 1: Total pneumococcal isolates found in different body fluids

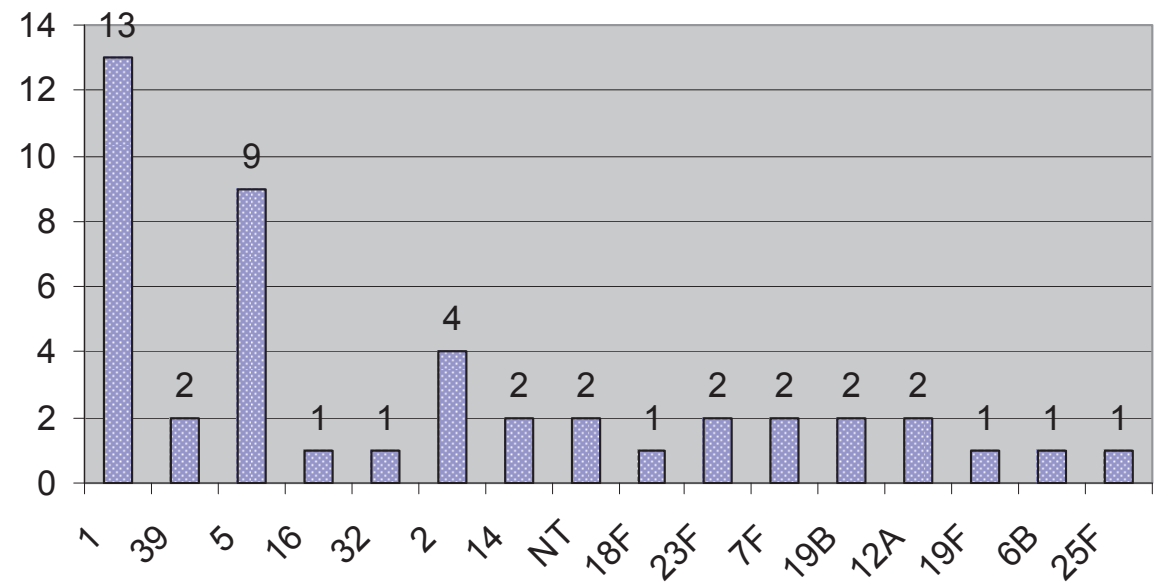

Fig 2: Distribution of different pneumococcal serotypes found in KCH during study (2004-2008)

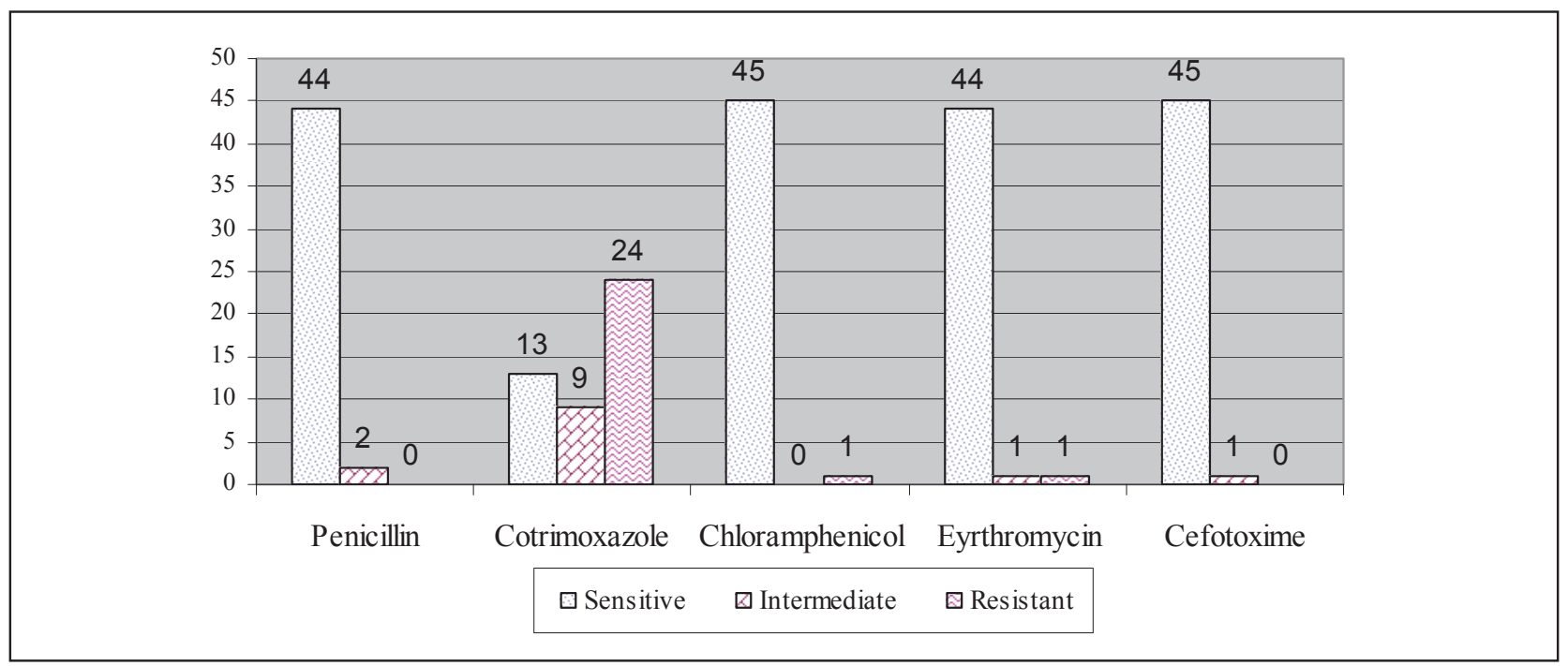

Fig 3: Antimicrobial resistance pattern and serotype distribution of invasive Streptococcus pneumoniae strains isolated from a Kanti Children Hospital in Nepal 


\section{Discussion}

The acute respiratory tract infection control program designed for developing countries by the World Health Organisation includes patient management protocols that recommend the use of an antimicrobial drug (Penicillin, Ampicillin, or Cotrimoxazole) for children with pneumonia ${ }^{13}$. The most remarkable finding of this study is the level of Cotrimoxazole resistance and the result was in accordance with the findings of Bangladesh ${ }^{13}$. Currently, the antibiotic resistance patterns of Streptococcus pneumoniae isolates vary widely from one country to another within Europe. Rates of resistance to penicillin have been reported to be increasing in some countries but resistance has remained at very low levels in other central European countries ${ }^{14,15}$. Studies have shown wide variations of antimicrobial resistance against Streptococcus pneumoniae among countries. None of the strains were resistant to penicillin and Cefotaxime in some countries including Hungary ${ }^{14}$. High level of resistance of Streptococcus pneumoniae to Penicillin has been reported from Asian countries like China, India, and the Philippines ${ }^{9}$. Chloramphenicol and Erythromycin resistance was observed in only 2. $17 \%$ of the strains without any relationship to Penicillin resistance. These drugs are the most common regimen used in the hospitals of our country, is still a good empirical choice for the treatment of pneumoccocal meningitis.

The increased rate of Cotrimoxazole resistance can be possibly be correlated with the wide use this antibiotic in the communities because of its dose convenience, cost effectiveness, and easy availability over the counter. Although multiple factors influence the emergence and spread of antimicrobial resistance, antimicrobial consumption is one of the most important. However, majority of Streptococcus pneumoniae in South Asia are now Cotrimoxazole resistant raising the question of whether WHO should shift from Cotrimoxazole to more expensive drugs for treatment ${ }^{16}$. This remarkable difference may have occurred because of the unlimited use of antibiotics, the lack of antibiotic policy and control in these countries, or as a result of the different antibiotic distribution policies ${ }^{13}$.

There were 16 serotypes isolated from the invasive pneumococcal infection of them one was nontypable. The most common serotype was 1 (27. 65\%) which was consistent with study done in Patan Hospital, $\mathrm{Nepal}^{17}$. Determination of the main serotypes that cause pneumococcal infection in each region of the world is of major importance in public health because of polyvalent conjugate vaccine formulations. Our results indicate that only four of the seven $(4,6 \mathrm{~B}, 9 \mathrm{~V}, 14,18 \mathrm{C}, 19 \mathrm{~F}$ and $23 \mathrm{~F}$ serotypes) most common serotypes of Streptococcus pneumoniae isolates from the Kanti Children Hospital are present in the $7-\mathrm{V}$ vaccine formulation. Worse, only five of the serotypes are present in both $9-\mathrm{V}$ vaccine (7$\mathrm{V}$ plus 1,5 serotypes) or $11-\mathrm{V}$ vaccine $(9-\mathrm{V}$ plus $3,7 \mathrm{~F}$ serotypes) formulations.

\section{Conclusion}

The study concluded that the high level of Cotrimoxazole resistance and also increasing resistance to other antimicrobial agents and wide serotype distribution of the Streptococcus pneumoniae isolates circulating in the population.

A more detailed study over a wider area and for a longer period on the pneumococcus on antimicrobial resistance and serotype distribution among the paediatric population of Nepal is needed. Such study may help to revise antimicrobial policy to treat invasive pneumococcal infections such as meningitis, pneumonia and septicaemia and for the development of an effective polyvalent conjugate vaccine for Nepal

\section{References}

1. Rajalakshmi B, Kanungo R. Cost effective method of serotyping S. pneumoniae using staphylococcal coagglutination. Indian J. Medical Microbiology. 2000;19(4):197-200.

2. Gratten M, Torzillo P, Morey F, Dixon J, Erlich $\mathrm{J}$, Hagger $\mathrm{J}$ et al. Distribution of Capsular Types and Antibiotic Susceptibility of Invasive Streptococcus pneumoniae Isolated from Aborigines in Central Australia. Journal of Clinical Microbiology. 1996;34(2):338-41.

3. Jesús O, Edurne L, Francisco JA, Fernando B, José C, Spanish Members of the European Antimicrobial Resistance Surveillance System. "Trends in antimicrobial resistance in 1, 968 invasive S. pneumoniae strains isolated in Spain hospitals (2001 to 2003): decreasing penicillin resistance in children's isolates", Journal of Clinical Microbiology. 2004;42(12):5571-7.

4. Brito DA, Ramirez M, Lencastre H. Serotyping Streptococcus pneumoniae by Multiplex PCR. Journal of Clinical Microbiology. 2003;41(6):2378-84.

5. Hofmann J, Cetron MS, Farley MM, Baughman WS, Facklam RR, Elliott JA, et al. The Prevalence of Drug-Resistant Streptococcus pneumoniae in Atlanta. New England Journal of Medicine. 1995;333(8):481-6.

6. Birtles A, Virgincar N, Sheppard CL, Walker RA, Johnson AP, Warner M, et al. Antimicrobial resistance of invasive Streptococcus pneumoniae isolates in a British district general hospital: the international connection. J Med Microbiol. 2004;53:1241-6. 
7. Wang $\mathrm{H}$, Huebner $\mathrm{R}$, Chen $\mathrm{M}$, Klugman K. Antibiotic Susceptibility Patterns of Streptococcus pneumoniae in China and Comparison of MICs by Agar Dilution and E-Test Methods. American Society for Microbiology. 1998;42(10):2633-6.

8. Muench DF, Rajnik M. Pneumococcal Infections. US: Department of Pediatrics, National Capital Consortium, Walter Reed Army Medical Center. 2008.

9. Alam MR, Saha SK, Nasreen T, Latif F, Rahman SR, Gomes DJ. Detection, Antimicrobial Susceptibility and Serotyping of Streptococcus pneumoniae from Cerebrospinal Fluid Specimens from Suspected Meningitis Patients. Bangladesh J Microbiol. 2007;24(1): 24-9.

10. NCCLS. Methods for dilution antimicrobial susceptibility tests for bacteria that grow aerobically, 5th ed. Approved standard M7-A6. Wayne, PA: National Committee for Clinical Laboratory Standards;2003.

11. NCCLS/CLSI. Performance standards for antimicrobial susceptibility testing; 15th informational supplement. CLSI document M100-S15. Wayne, PA: Clinical and Laboratory Standards Institute; 2005.

12. NCCLS. Performance standards for antimicrobial susceptibility testing; 14th informational supplement. NCCLS document M100-S14. Wayne, PA: NCCLS;2004.

13. Saha SK, Rikitomi N, Ruhulamin M, Masaki $\mathrm{H}$, Hanif M, Islam M, et al. Antimicrobial Resistance and Serotype Distribution of Streptococcus pneumoniae Strains Causing Childhood Infections in Bangladesh, 1993 to 1997. Journal of Clinical Microbiology. 1999; 37(3):798-800.

14. Dobay O, Rozgonyi F, Hajdú E, Nagy E, Knausz M, Amyes SGB. Antibiotic susceptibility and serotypes of Streptococcus pneumoniae isolates from Hungary Journal of Antimicrobial Chemotherapy. 2003;51;887-93.

15. Michel N, Watson M, Baumann F, Perolat P, Garin B. Distribution of Streptococcus pneumoniae Serotypes Responsible forPenicillin Resistance and the Potential Role of New ConjugateVaccines in New Caledonia. Journal of clinical microbiology. 2005;43(12):6060-3.

16. Zaidi AKM. Resistant respiratory infections threaten developing countries. The APUA Newsletter. 2003;21(3):1-2.

17. Williams EJ, Thorson S, Maskey M, Mahat S, Hamaluba M, Dongol S, et al. Hospital based surveillance of invasive pneumococcal disease among young children in urban Nepal. Clinical Infectious diseases. 2009;48(24):167. 